\title{
Botulinumtoxin-Therapie in der Neurologie: Indikationen, Technik und Ultraschallsteuerung
}

\author{
Botulinum Toxin Treatment in Neurology: Indications, \\ Technique and Ultrasound-Guidance
}

VNR

\section{9}

Bibliografie

DOI http://dx.doi.org/

$10.1055 / \mathrm{s}-0035-1552683$

Klin Neurophysiol 2015; 46:

56-64

(c) Georg Thieme Verlag KG

Stuttgart · New York

ISSN 1434-0275

Korrespondenzadresse

Prof. Dr. Uwe Walter

Klinik und Poliklinik für

Neurologie

Universitätsmedizin Rostock

Gehlsheimer Straße 20

18147 Rostock

uwe.walter@med.uni-rostock.

de

U. Walter ${ }^{1}$, D. Dressler ${ }^{2}$

Klinik und Poliklinik für Neurologie, Universitätsmedizin Rostock

${ }^{2}$ Sektion Bewegungsstörungen, Klinik für Neurologie und Klinische Neurophysiologie, Medizinische Hochschule Hannover

\section{Einleitung}

Botulinumtoxin (BT) blockiert hoch selektiv und sehr effektiv die cholinerge synaptische Transmission an der Endplatte der Muskulatur und führt nach lokaler Injektion zur teilreversiblen Parese der Muskeln. BT blockiert über den synaptischen Mechanismus auch die Sekretion exokriner Drüsen sehr effektvoll. Die Wirkung von BT beginnt einige Tage nach der lokalen Injektion, erreicht das Maximum der Wirkung innerhalb von 1-2 Wochen und klingt nach 2-3 Monaten in der Regel wieder ab. Dieser Effekt wird in der Literatur bei vielen Anwendungen beschrieben und stellt die Indikationsgrundlage zur fokalen Behandlung spezifischer Krankheitsbilder dar [1]. Die höchsten Anwendungszahlen erfährt BT allerdings in der Schönheitsmedizin.

In der Neurologie wird BT häufig eingesetzt für die Behandlung der Syndrome hyperaktiver Muskeln, wie z.B. bei Spastik nach Schlaganfall oder bei frühkindlichen Zerebralparesen, Dystonien, dem Spasmus hemifacialis, fazialen Synkinesien, Tics und manchen Tremores. Weitere BT-Anwendungen sind zur therapeutischen Schwächung normaler Muskulatur im Kontext von Engpasssyndromen beschrieben, um betroffene Nerven zu dekomprimieren (z.B. neurogenes Thoracicoutlet-Syndrom, Piriformis-Syndrom) oder anatomische Besonderheiten zu behandeln (z.B. Omohyoid-Muskel-Syndrom) [2-4]. Die Behandlung hat entweder eine Funktionsverbesserung (z.B. bei Schreibkrampf, moderater Spastik) oder eine Schmerzreduktion und Erleichterung der Pflege

Häufige neurologische Indikationen
zur BT-Behandlung
Dystonien:
kraniale (Blepharospasmus, oromandi-
buläre, periorale) Dystonie
zervikale Dystonie
pharyngeale Dystonie
- spasmodische Dysphonie
Schreibkrampf
- Musiker-Dystonie
- segmentale Dystonien
Periphere Dyskinesien:
- Spasmus hemifacialis
- Reinnervations-Synkinesien
Spastik:
- Schlaganfall mit Spastik in Nacken, Schulter,
Arm, Hand, Fingern, Becken, Knien, Fußge-
lenken, Großzehe und Zehen
- Zerebralparese, Querschnittssyndrome
Weitere Indikationen:
- chronische Migräne
- Bruxismus
- tardive Dyskinesie
Tremor
Drüseninjektion:
Hyperhidrosis
vermehrte Tränensekretion
Tyics

(z.B. Handöffnung, Reduktion einer Adduktorenspastik) zum Ziel. Ein weiteres Indikationsgebiet für BT ist die Behandlung exokriner Drüsen z.B. bei Hyperhidrose, Hypersalivation mit Aspirationsgefahr oder sog. Krokodilstränen. Eine neue Zulassung für BT ist die therapieresistente chronische Migräne [5].

BT muss direkt in das Zielgewebe injiziert werden. Daher ist die exakte Positionierung der Injektionsnadel relevant, um den richtigen Muskel oder das Drüsengewebe zu „treffen“ und damit den maximalen Wirkstoffeffekt bei minimalen 
Nebenwirkungen mit einer geringen Dosis zu erzielen.

Die Botulinumtoxin-Behandlung in der Neurologie soll eine Funktionsverbesserung, eine Schmerzreduktion, eine Risikoreduktion (z. B. der Aspiration) oder eine Pflegeerleichterung bewirken.

\section{Konventionelle Methode der BT-Injektionstechnik}

Klinische Inspektion und anatomische Landmarken der Muskelbehandlung

Die Indikation für BT wird nach dem klinischen Befund entsprechend dem Krankheitsbild und der Funktionalität gestellt. Die klinische Untersuchung beruht auf der Inspektion und der Palpation des Zielmuskels. Durch aktive oder passive Bewegungen wird der Muskel am Sehnenverlauf identifiziert. Nützlich zur Identifikation der Zielmuskeln ist die Orientierung an anatomischen Landmarken. Die Bewegung der Injektions- oder EMG-Nadel bei passiver Bewegung des Zielmuskels kann ebenso helfen, die richtige Injektionsstelle zu sichern. Diese Methoden stoßen dann an ihre Grenzen, wenn tiefere Muskeln unter meist pathologischen Bedingungen (Atrophie, massive Spastik) identifiziert werden müssen. Am Unterarm stellen folgende Muskeln immer eine Herausforderung dar: M. flexor carpi radialis, M. pronator teres, M. flexor digitorum superficialis II/III und der M. flexor pollicis longus [6]. In einer klinischen Studie an 226 Kindern mit frühkindlicher Spastik wurde die Trefferquote der BTInjektion durch inspektorische und palpatorische Identifikation des Muskels mit EMG-gesteuerter Injektion verglichen. Die Quote für die 1. Gruppe war deutlich niedriger für Unterarmmuskeln (13\% versus $35 \%$ ), den medialen Kopf des M. gastrocnemius (46\%) und den M. tibialis posterior (11\%) [7]. Bei Erwachsenen ergab sich eine erstaunlich hohe Fehlinjektionsrate von 57\% für den lateralen Kopf des M. gastrocnemius [8, 9]. Demgegenüber scheint die rein klinische Methode bei den großen und gut zugänglichen Muskeln in der Behandlung der zervikalen Dystonie und der Adduktorenspastik völlig ausreichend zu sein $[10,11]$.

Die EMG-kontrollierte Botulinumtoxin-Injektion kann gerade in tieferen Muskelschichten zu einer deutlich gezielteren Applikation des Wirkstoffs beitragen.

\section{EMG- und kontrastmittelunterstützte} BT-Injektion

Überaktive Muskeln und deren Funktionsstörung können elektrophysiologisch mittels EMG identifiziert werden. Durch die Verwendung von spe- ziellen, elektrisch isolierten Injektionsnadeln kann gleichzeitig das EMG abgeleitet und so die BT-Applikation verbessert werden [12]. Jedoch gibt es Einschränkungen der EMG-geführten Technik, da der Zielmuskel häufig nicht alleine überaktiv ist und Patienten mit hochgradigen spastischen Paresen die Muskulatur nicht selektiv innervieren können. Gerade Patienten mit Dystonie bzw. Spastik zeigen typischerweise eine Koaktivierung aller Umgebungsmuskeln [10]. Die EMG-gesteuerte Injektion kann durch eine fokale elektrische Stimulation des Muskels verbessert werden (aktive EMG-Steuerung) [12]. Nachteile der EMG-gesteuerten Injektion sind die relativ großen und schmerzhaften Nadeln, die potenzielle Schädigung benachbarter Nerven und Gefäße und die fehlende Kontrolle über den Injektionsort. Die EMG-Methode ist zur Injektion exokriner Drüsen natürlich ungeeignet.

Für besondere Indikationen ist eine Kombination von EMG und Fluoroskopie beschrieben worden, um z.B. die Injektion in den M. piriformis bei Piriformis-Syndrom, in den M. scalenus anterior und M. scalenus medius beim neurogenen Thoracicoutlet-Syndrom und in den M. longus colli bei Antecollis zu optimieren [13-15]. Die Fluoroskopie ist aber mit einer Strahlenbelastung und intramuskulärer Kontrastmittel-Injektion verbunden.

\section{Bildgebungskontrolle der Injektion mit CT und MRT}

Computertomografie (CT) und Magnetresonanztomografie (MRT) ermöglichen eine hochauflösende Darstellung von Muskulatur, Drüsen und benachbarten anatomischen Strukturen. Die kombinierte Injektion von Kontrastmittel und BT erlaubt eine sehr genaue Dokumentation der Injektionsstelle, allerdings nicht in Echtzeit [1618]. In einer prospektiven klinischen Studie an 146 CT-gesteuerten BT-Injektionen in den M. scalenus anterior wurde eine nicht vernachlässigbare Komplikationsrate berichtet, darunter die transiente Plexusblockade $(3,4 \%)$ und das HornerSyndrom (0,7\%) [19]. Darüber hinaus schlägt neben einer relevanten Strahlenbelastung eine hohe zeitliche Personalbindung für diese Behandlung zu Buche, die damit insgesamt aufwendig und kostenintensiv ist.

\section{Ultraschallgeführte Injektion}

$\nabla$

Im B-Bild des Ultraschalls (US) ist es möglich, nicht invasiv und in Echtzeit sowohl Muskulatur und exokrine Drüsen als auch deren anatomische Umgebungsstrukturen darzustellen. Damit erlaubt US eine kontinuierliche Injektionskontrolle während der gesamten Behandlung. Die US-geführte BT-Injektion wurde erstmalig von japanischen Neurologen und später durch deutsche Neuropädiater beschrieben [20, 21]. Die US-Anwendung erfordert genaue anatomische Kennt-

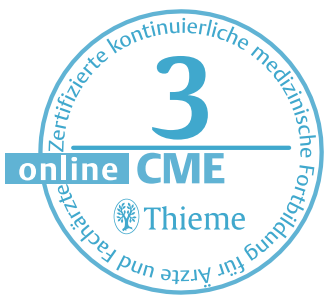


nisse über die Lokalisation der Drüsen und der Muskeln, die mittels anatomischer Lehrbücher zu erarbeiten bzw. zu wiederholen sind und ggf. an Leichen oder Phantomen vertieft werden können [22]. Hierfür werden zahlreiche Kurse und internetbasierte Anleitungen angeboten.

\section{Digitale Angebote}

Adressen für Internet-Kurse:

- www.munichultrasoundcourse.com/

- www.theonlinelearningcenter.com/ultra sound/musculoskeletal-ultrasound-cme

- theultrasoundsite.co.uk/category/ resources/guided-injections/

Mobile Learning-Apps:

- www.imedicalapps.com/2013/01/mucapp-msk-ultrasound-guided-interventionap/

- en.knicket.com/details/pnyt/android/sonoguide

- itunes.apple.com/de/app/sonoguide/ id $470488939 ? \mathrm{mt}=8$

\section{Technische Voraussetzungen}

für den Ultraschall

Die konventionellen US-Systeme, die üblicherweise in der Neurologie genutzt werden, um z.B. den extrakraniellen Gefäßstatus zu erheben, sind in der Regel für Muskelsonografie oder Drüsendarstellung ausreichend. Im B-Bild der Linearsonden erreichen Schallfrequenzen um $6-12 \mathrm{MHz}$ ausreichende Gewebetiefen zur Darstellung der Muskeln und Drüsen, z.B. im Nacken, Bein oder Rücken, während höhere Schallfrequenzen (10$18 \mathrm{MHz}$ ) eine bessere Bildqualität für oberflächliche Muskeln bieten [23]. Die „Thorax-AbdomenSonden“" mit Grundfrequenzen um 2-3 MHz sind weniger geeignet. Die Hersteller empfehlen für ihre Geräte meist eine vorgegebene Grundeinstellung bzgl. der Bildrate und -verarbeitungsfunktion. Zur optimalen Muskeldarstellung wird eine rechtwinklige Sondenstellung zur Haut mit nur leichtem Druck auf das unterliegende Gewebe empfohlen. Die Gewebeechogenität hängt sowohl vom Beschallungswinkel als auch vom Druck auf das Gewebe ab. Die Ausrichtung der Sondenposition wird gemäß Richtlinien der DEGUM wie folgt empfohlen: Die linke Seite auf dem Bildschirm entspricht der medialen bzw. ulnaren oder kranialen bzw. proximalen Seite der untersuchten Körperregion [24]. Abweichend von dieser Konvention kann auch ein eigener Laborstandard festgelegt werden bzw. nimmt man im Einzelfall eine entsprechende Beschriftung im B-Bild vor.

Für die US-geführte BT-Injektion sind Linearsonden mit Schallfrequenzen von $6-12 \mathrm{MHz}$ geeignet.

\section{Ultraschallbildgebung der Muskeln}

Ein normaler Muskel imponiert im US-B-Querschnittsbild wie ein „Sternenhimmel“ und im longitudinalen Schnittbild als gefiederte Struktur. Das Aussehen verändert sich bei Kontraktion des Muskels teils erheblich [22, 23]. Das Muskelgewebe ist vornehmlich schwach echogen; die Echogenität steigt mit zunehmender Fibrose, Degeneration oder Verfettung [25-27]. Eine Größen- bzw. Durchmesserabschätzung gelingt im Allgemeinen gut mittels US für Extremitätenmuskeln, paraspinale Muskulatur, kraniale und Gesichtsmuskulatur [28-31]. Einzelne Muskelfibrillen können im US nicht aufgelöst werden, während die Muskelbündel (Faszikel), die vom Bindegewebe umschlossen sind (Perimysium), mit den hoch auflösenden, hoch frequenten Linearsonden hyperechogen darstellbar sind. Das relativ dicke Epimysium erlaubt die Unterscheidung verschiedener Muskeln [23]. Abb. 1 zeigt ein Beispiel für die US-Darstellung normaler Muskeln. Durch zusätzliche Dopplereigenschaften können im Farbduplex-Modus auch die Gefäße dargestellt werden.

Der normale gesunde Muskel zeigt im Ultraschall ein schwach echogenes Muster mit typischer Fiederung.

\section{Ultraschallbildgebung der Speicheldrüsen}

Mittels US-B-Bild sind Speicheldrüsen gut abbildbar [32]. Während bereits durch Palpation der oberflächliche Anteil der Glandula parotis gut identifizierbar ist, gelingt dies für den retromandibulären Anteil oder die Glandula submandibularis nur in $30 \%$ der Fälle. Im Ultraschall sind Glandula parotis und Glandula submandibularis ähnlich wie das Schilddrüsengewebe als homogene Textur mit leichter Hyperechogenität gut vom Umgebungsgewebe differenzierbar ( $\bullet$ Abb.2).

\section{Ultraschallgeführte BT-Injektion}

$\nabla$

\section{Allgemeine Informationen}

BT-Injektionen werden gewöhnlich mit einer $20 \mathrm{~mm}$ - (27 Gauge; Außendurchmesser: 0,40 $\mathrm{mm}$ ) oder $40 \mathrm{~mm}$-Nadel (27 Gauge) durchgeführt. Für tiefere Muskelschichten werden $80 \mathrm{~mm}$ - (23 Gauge; 0,60 mm) oder sogar $120 \mathrm{~mm}$-Nadeln (25 Gauge; $0,50 \mathrm{~mm}$ ) benutzt. Sehr tief gelegene Muskeln ( $>6 \mathrm{~cm}$ unter Hautoberfläche) sind im Allgemeinen ungeeignet für die ultraschallgesteuerte BT-Injektion. Für die Hautdesinfektion sollte eine alkoholfreie Lösung benutzt werden, um die empfindliche gummierte Schutzschicht der USSonde und damit die Ultraschallkristalle nicht zu beschädigen. Das gewöhnliche handelsübliche US-Gel hat keine nachweislichen Auswirkungen auf die Infektionsrate oder Hautreaktionen. Ein besonderes bakteriostatisch wirksames US-Gel ist nicht notwendig [33]. Wir empfehlen aber spe- 
ziell bei der Injektion tiefer Muskeln (Tiefe $>2 \mathrm{~cm}$ ) entweder das Abwischen des Gels und die nochmalige Desinfektion der Injektionsstelle vor der Nadelinsertion oder die Verwendung eines sterilen Gels. Selbstverständlich ist nach jedem Gebrauch die US-Sonde gemäß den geltenden Hygienestandards zu reinigen und zu desinfizieren. Prinzipiell sind 2 US-geführte Injektionstechniken möglich: Die „In-plane“- und die „Off-plane“Technik. Bei der „In-plane“-Technik wird die Injektionsnadel seitlich neben der US-Sonde positioniert und die Nadel in ihrem gesamten Verlauf in der Bildebene dargestellt und geführt. Für diese Technik gibt es z.T. spezielle Nadelhalterungen an der Sonde, die vorwiegend für Biopsien benutzt werden [34]. Nach unserer Erfahrung ist die „Offplane“-Technik mit freihändiger Nadelführung in aller Regel für die BT-Injektion ausreichend. Hierfür wird die Nadel in der Mitte über (oder unter) der US-Sonde platziert - dabei wird die Nadelspitze erst bei Erreichen der Zielstruktur im USBild sichtbar. Die US-Sonde sollte dabei so positioniert sein, dass die Zielstruktur in der Bildmitte liegt ( $\bullet$ Abb. 1, 2). Damit hat man durch die Sondengeometrie in Relation zur Haut eine Orientierungshilfe für die Nadelführung. Durch Druck der noch bekappten Nadel auf die Haut („Anklopfmanöver“) mit resultierenden Bewegungsartefakten bzw. die Bewegungsartefakte der eingeführten Nadel kann man die Nadelposition im US-Bild kontrollieren [33, 35]. Die Nadel selbst wird im Ultraschallbild als dunkler Strich dargestellt, abhängig vom Nadeldurchmesser. Die Nadelspitze ist oft nur indirekt erkennbar. Bei Verwendung ultraschall-optimierter Injektionsnadeln ist die Nadel kräftig echogen, mit der „In-plane“-Technik z.T. sogar als Doppellinie, sichtbar. Die injizierte BT-Lösung kann als echoarmes Depot im Bild dokumentiert werden, dies ergibt dann einen Nachweis der Lokalisation und des Injektionsvolumens. Wir empfehlen neben einem Ausdruck des US-Bildes (Zielstruktur, BT-Depot) auch eine schriftliche Dokumentation der verwendeten Nadelgröße, die Art der verwendeten US-Sonde und eine Anmerkung, wie der Patient die Prozedur vertragen hat $[35,36]$.

Die Technik der US-gesteuerten Injektion sollte zu Beginn am Phantom bzw. an einem im Lebensmittelhandel erworbenen Fleischstück trainiert werden. Dann sollte sukzessive die „Auge-Hand“Koordination anhand der Behandlung zunächst größerer Muskeln verbessert werden [35]. Der Einsatz von US zur BT-Injektion erfordert in der Regel einen höheren Zeitbedarf als die Injektion nach rein klinischen Kriterien („Aus-der-Hand“Methode). Bei ausreichender Erfahrung wird dieser zusätzliche Zeitaufwand jedoch kleiner, sodass die US-geführten Injektion in größeren BTAmbulanzen zunehmend eingesetzt wird [37].

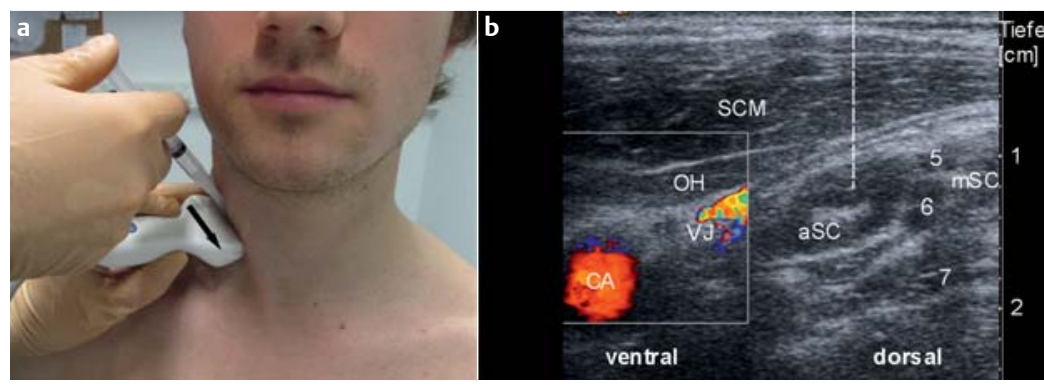

Abb. 1 Ultraschall-geführte BT-Injektion in der lateralen Halsregion. a) Position der Ultraschallsonde und der Nadel bei Anzielung des M. scalenus anterior („Off-plane“-Injektionstechnik). Der Pfeil gibt die Richtung der Sonde an (Pfeilspitze entspricht der linken Seite des B-Bildes in [b]). b) B-Bild zeigt den M. sternocleidomastoideus (SCM), M. omohyoideus (OH), M. scalenus anterior (aSC) und M. scalenus medius (mSC). CA = A. carotis communis; VJ=Vena jugularis interna; 5, 6, 7 = 5., 6., 7. zervikale Nervenwurzeln. Die Haut ist hyperechogen zu erkennen,

das Fettgewebe hat eine mittlere Echogenität. Die gestrichelte Linie ist der mutmaßliche Injektionsweg der Nadel in den M. scalenus anterior.

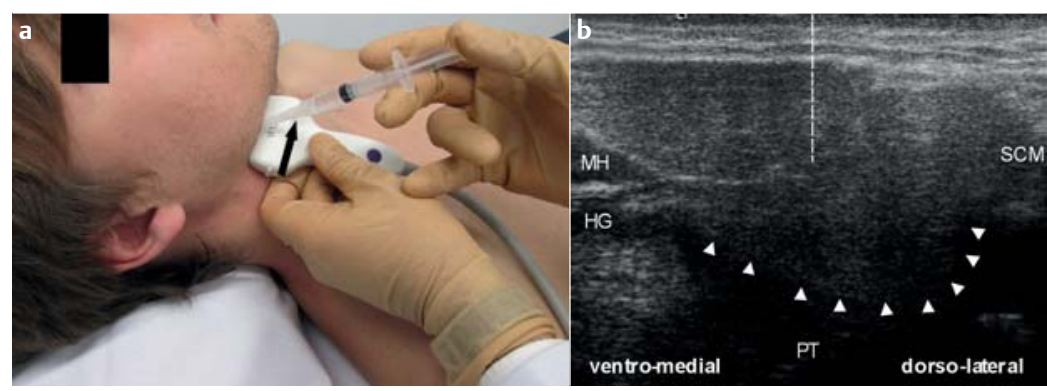

Abb.2 Ultraschall-geführte BT-Injektion in die Glandula submandibularis. a) Position der Ultraschallsonde und der Nadel für die Injektion in die Glandula submandibularis. Der Pfeil gibt die Richtung der Sonde an (Pfeilspitze entspricht der linken Seite des B-Bildes in [b]). b) B-Bild der Gl. submandibularis mit ihren Nachbarstrukturen: $\mathrm{HG}=\mathrm{M}$. hyoglossus; $\mathrm{MH}=\mathrm{M}$. myohyoideus; PT = Tonsilla palatina; SCM = M. sternocleidomastoideus. Die gestrichelte Linie zeigt den erwarteten Injektionskanal.

\section{Intramuskuläre BT-Injektion}

Die Empfehlungen der Autoren zur Anwendung der US-Führung bei der BT-Injektion in die muskulären und glandulären Zielstrukturen sind in - Tab. 1 zusammengestellt. Einige Muskelgruppen sind nicht (M. pterygoideus) oder nur eingeschränkt für die US-geführte BT-Injektion geeignet (Gesichtsmuskulatur, Pharynxmuskeln, Larynxmuskeln) [31, 38, 39]. Die laryngealen Muskeln (M. vocalis, M. cricoarythenoideus posterior) können transoral bei Verwendung eines Endoskops oder transdermal injiziert werden, wobei hier eher die EMG-geführte Methode empfohlen wird [40, 41]. Die supra- and infrahyoidale Muskulatur ist im US gut sichtbar [38, 42], wobei hier nur selten eine BT-Behandlungsindikation besteht. Die US-geführte Applikation ist empfehlenswert für die laterale und dorsale Nackenmuskulatur, Schultermuskulatur, proximale und distale Armmuskulatur, Handmuskulatur und generell an der unteren Extremität. Vor allem der Einsatz von US zur Injektion spastischer, schon umgebauter Muskelgruppen ist sehr hilfreich. Bei Kindern mit spastischer Zerebralparese sollte laut einer europäischen Konsensus-Empfehlung die BT-Applikation prinzipiell EMG- oder US-ge- 


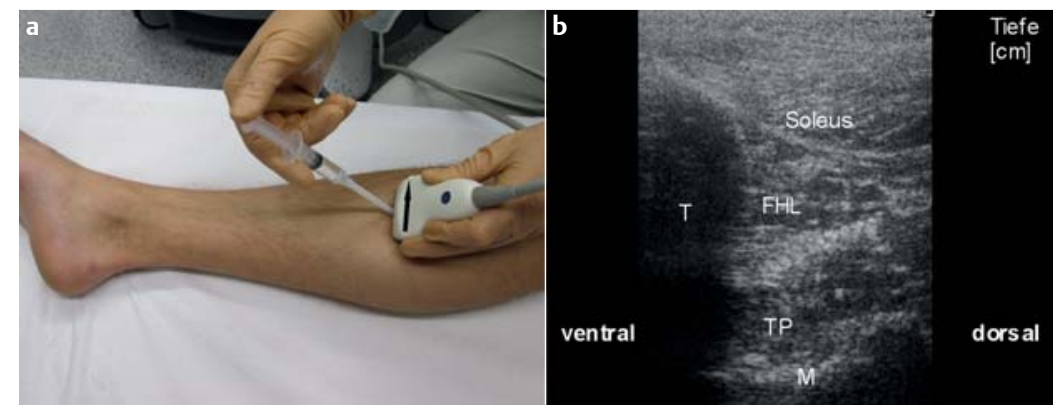

Abb.3 Ultraschall-geführte BT-Injektion in Beinmuskeln. a) Position der Sonde und der Injektionsnadel („Off-plane“-Technik) zur geplanten Injektion in den M. tibialis posterior. Der Pfeil gibt die Richtung der Sonde an (Pfeilspitze entspricht der linken Seite des B-Bildes in [b]). b) Das B-Bild zeigt den M. soleus, M. flexor hallucis longus (FHL) und M. tibialis posterior (TP), die Tibia (T) und die Membrana interossea (M).

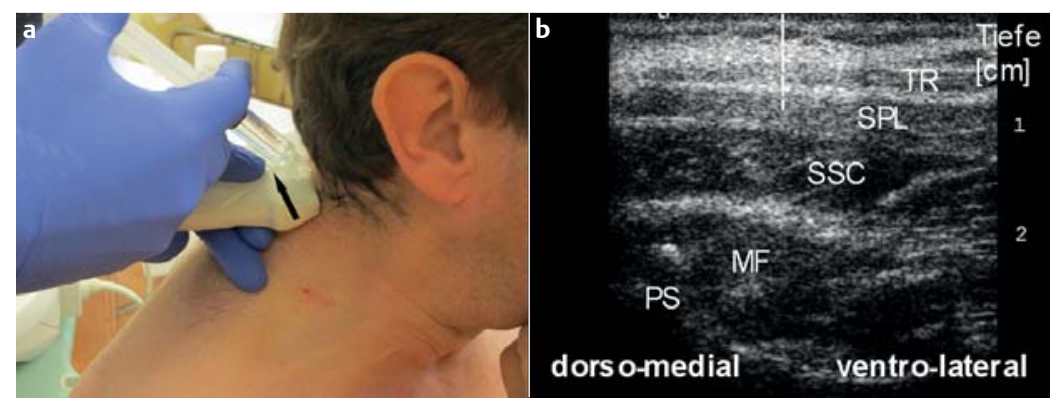

Abb.4 Ultraschall-geführte BT-Injektion in der Nackenregion. a) Position der Ultraschallsonde und der Nadel bei Anzielung des M. splenius capitis. Der Pfeil gibt die Richtung der Sonde an (Pfeilspitze entspricht der linken Seite des B-Bildes in [b]). b) B-Bild des M. trapezius (TR), M. splenius capitis (SPL), M. semispinalis capitis (SSC), M. multifidus (MF); PS= Processus spinosus. Die gestrichelte Linie zeigt den erwarteten Injektionskanal.

führt erfolgen [43]. Die Akzeptanz bei Kindern ist höher für die US-geführte Methode.

Die US-geführte Technik hat den Vorteil, dass dem Zielmuskel anliegende Nerven und Gefäße visualisiert und damit potenzielle Nebenwirkungen durch Fehlinjektion verringert werden können.

Das gilt insbesondere für Behandlung des M. scalenus anterior, des M. longus colli, der Unterarmmuskeln, des M. psoas und der Beinmuskeln ( $\bullet$ Abb. 1, 3) [44-48]. Besonders dünne Muskeln wie z. B. der M. omohyoideus oder der M. longissimus capitis können nur richtig mittels US identifiziert werden ( $\bullet$ Abb.1). Die Nebenwirkung der Dysphagie nach BT-Injektion in den M. sternocleidomastoideus konnte signifikant durch die USgeführte Injektionstechnik reduziert werden [49]. Eine kleine prospektive randomisierte Studie verglich die US-geführte und die EMG-geführte Injektion mit der konventionellen BT-Applikation zur Reduktion der Spastik im Unterarm bei Schlaganfallpatienten [50]. Diese ergab eine klare Überlegenheit der US-geführten Methode. Die Überlegenheit von US-geführter über die rein manuelle Injektionstechnik zur Reduktion einer Armspastik nach Schlaganfall konnte kürzlich in einer weiteren randomisierten Studie bestätigt werden [51].
Tab. 1 Graduierte Empfehlungen zur US-Führung der BTInjektion in verschiedene muskuläre and glanduläre Zielstrukturen.

\begin{tabular}{|c|c|}
\hline $\begin{array}{l}\text { Empfehlungs- } \\
\text { grad der US- } \\
\text { Führung }\end{array}$ & Zielstrukturen \\
\hline Niedrig ${ }^{1}$ & $\begin{array}{l}\text { mimische Muskeln² } \\
\text { supra- und infrahyoidale Muskeln² } \\
\text { pharyngeale Muskeln } \\
\text { M. temporalis } \\
\text { M. masseter } \\
\text { M. sternocleidomastoideus } \\
\text { M. trapecius } \\
\text { Schultermuskeln } \\
\text { proximale Armmuskeln } \\
\text { intrinsische Handmuskeln } \\
\text { oberflächliche paravertebrale Muskeln } \\
\text { oberflächliche abdominelle Muskeln } \\
\text { glutäale Muskeln } \\
\text { Oberschenkel-Adduktoren } \\
\text { M. tibialis anterior } \\
\text { Glandula sublingualis } \\
\text { Glandula lacrimalis² } \\
\text { Schweißdrüsen }\end{array}$ \\
\hline Intermediär & $\begin{array}{l}\text { M. splenius capitis } \\
\text { M. semispinalis capitis } \\
\text { M. levator scapulae } \\
\text { ischiokrurale Muskeln } \\
\text { M. gastrocnemius } \\
\text { Glandula parotis }\end{array}$ \\
\hline Hoch $^{5}$ & $\begin{array}{l}\text { M. longus colli } 3,4 \\
\text { M. longus capitis } \\
\text { laryngeale Muskeln }^{3,4} \\
\text { M. psoas (inguinaler Anteil) } \\
\text { M. psoas (spinaler Anteil) } \\
\text { M. obliquus capitis inferior } \\
\text { M. scalenus anterior } \\
\text { M. scalenus intermedius } \\
\text { M. omohyoideus } \\
\text { M. longissimus capitis } \\
\text { M. longissimus cervicis } \\
\text { einzelne Unterarmmuskeln } \\
\text { einzelne Fingerflexoren } \\
\text { einzelne Fingerextensoren } \\
\text { M. gracilis } \\
\text { M. soleus } \\
\text { M. tibialis posterior } \\
\text { M. extensor hallucis longus } \\
\text { Glandula submandibularis }\end{array}$ \\
\hline
\end{tabular}

1 niedriger Empfehlungsgrad wegen einfacher visueller/manueller Anzielung bzw. wegen geringer Fallzahlen

${ }^{2}$ technisch schwierig wegen der geringen Größe der Zielstruktur

${ }^{3}$ technisch schwierig wegen der tiefen Lage der Zielstruktur

${ }^{4}$ technisch schwierig wegen gefährdeter Nachbarstrukturen

${ }^{5}$ hoher Empfehlungsgrad bei Dystonie, milder Spastik, infantiler Zerebralparese

${ }^{6}$ beachte die korrekte Injektionstechnik

${ }^{7}$ bei M.-omohyoideus-Syndrom

Eine ähnliche Studie verglich die US-geführte Applikation mit der rein manuellen Injektion und EMG-geführter Injektion in den lateralen und medialen Kopf des M. gastrocnemius bei Erwachsenen mit spastischem Pes equinovarus nach Schlaganfall [52]. Diese zeigte ebenfalls eine Überlegenheit der technischen Anwendung. Die EMG- oder US-geführte Injektionsmethode (oder deren Kombination) ist besonders erfolgreich bei 
der Behandlung der Unterarmmuskulatur bei tätigkeitsbezogener Dystonie [53]. Bei vielen unserer Patienten mit Schreibkrampf konnte die BTDosis durch Anwendung der US-geführten Injektion reduziert werden.

Bei Kindern mit spastischer Zerebralparese sollte prinzipiell die intramuskuläre BT-Applikation mit Unterstützung des EMG oder des US erfolgen. Bei milder Armspastik und bei tätigkeitsbezogener Dystonie ermöglicht die US-geführte BT-Injektion durch die resultierende Standardisierung der Therapie einen reproduzierbaren Funktionsgewinn.

\section{Intraglanduläre BT-Applikation}

Die US-Führung der BT-Injektion in die Glandula submandibularis ist empfehlenswert ( $\mathbf{A b b} \mathbf{2}$ ). Die retromandibuläre Portion der Glandula parotis lässt sich mittels US ebenfalls besser anzielen und sich somit die gefürchtete Nebenwirkung einer Fehlinjektion in die parapharyngeale Muskulatur mit resultierender Schluckstörung vermeiden. In kleinen Studien wurde eine Überlegenheit der US-geführten Injektion zur Behandlung der Sialorrhö beim Parkinson-Syndrom berichtet [54]. Gute Therapieerfolge wurden auch für die Behandlung der Hypersalivation mittels US-geführter BT-Injektion in die Glandula submandibularis und Glandula parotis bei Kindern und Erwachsene mit verschiedenen neurologischen Erkrankungen publiziert [55-59]. Die Glandula sublingualis und die Glandula lacrimalis können ebenfalls mittels US dargestellt werden, sind aber in der Praxis weniger relevant $[32,60]$.

\section{Zusammenfassung}

- Die Therapie mit Botulinumtoxin (BT) wird in der Neurologie zur lokalen Behandlung von hyperaktiven Muskeln und zur Blockierung von exokrinen Drüsen eingesetzt.

- Typische BT-Indikationen sind die Dystonie, die Spastik, Reinnervations-Synkinesien, Engpasssyndrome, Hyperhidrose und Hypersalivation.

- Um eine optimale Behandlung mit hoher Effektivität und geringen Nebenwirkungen zu erzielen, sind die richtige Indikationsstellung, adäquate BT-Dosis und die exakte BT-Applikation im Zielgewebe entscheidend.

- Die Ultraschall-Bildgebung (US) ist eine nichtinvasive Methode zur Echtzeit-Darstellung von Muskeln und exokrinen Drüsen.

- Mittels US kann die Injektion von Substanzen unter Sicht gesteuert werden.

- Die US-geführte Injektion ergibt eine Qualitätsverbesserung der BT-Behandlung.
Interessenkonflikt: U. Walter erhielt Vortragshonorare von Ipsen und Merz Pharma und Reisekosten-Erstattung von Allergan.

D. Dressler erhielt Beraterhonorare von Allergan, Ipsen, Merz, Eisai, Addex und Syntaxin Pharma.

\section{Literatur}

1 Dressler $D$. Clinical applications of botulinum toxin. Curr Opin Microbiol 2012; 15: 325-336

2 Jankovic $D$, Peng P, van Zundert A. Brief review: Piriformis syndrome: etiology, diagnosis, and management. Can J Anesth 2013; 60: 1003-1012

3 Torriani M, Gupta R, Donahue DM. Botulinum toxin injection in neurogenic thoracic outlet syndrome: results and experience using a ultrasound-guided approach. Skeletal Radiol 2010; 39: $973-980$

4 Su PH, Wang TG, Wang YC. Ultrasound-guided injection of botulinum toxin in a patient with omohyoid muscle syndrome: a case report. J Clin Ultrasound 2013; 41: $373-376$

5 Aurora SK, Winner P, Freeman MC et al. OnabotulinumtoxinA for treatment of chronic migraine: pooled analyses of the 56-week PREEMPT clinical program. Headache 2011; 51: 1358-1373

6 Henzel MK, Munin MC, Niyonkuru C et al. Comparison of surface and ultrasound localization to identify forearm flexor muscles for botulinum toxin injections. PM R 2010; 2: 642-646

7 Chin TY, Nattrass GR, Selber $P$ et al. Accuracy of intramuscular injection of botulinum toxin $A$ in juvenile cerebral palsy: a comparison between manual needle placement and placement guided by electrical stimulation. J Pediatr Orthop 2005; 25: 286-291

8 Picelli A, Bonetti P, Fontana $C$ et al. Accuracy of botulinum toxin type $A$ injection into the gastrocnemius muscle of adults with spastic equinus: manual needle placement and electrical stimulation guidance compared using ultrasonography. J Rehabil Med 2012; 44: 450-452

9 Schnitzler A, Roche N, Denormandie P et al. Manual needle placement: accuracy of botulinum toxin A injections. Muscle Nerve 2012; 46: 531-534

10 Jankovic J. Needle EMG guidance for injection of botulinum toxin. Needle EMG guidance is rarely required. Muscle Nerve 2001; 24: 1568-1570

11 Mall V, Heinen F, Siebel $A$ et al. Treatment of adductor spasticity with BTX-A in children with CP: a randomized, double-blind, placebo-controlled study. Dev Med Child Neurol 2006; 48: 10-13

12 Barbano RL. Needle EMG guidance for injection of botulinum toxin. Needle EMG guidance is useful. Muscle Nerve $2001 ; 24: 1567-1568$

13 Childers MK, Wilson DJ, Gnatz SM et al. Botulinum toxin type $A$ use in piriformis muscle syndrome: a pilot study. Am J Phys Med Rehabil 2002; 81: 751 - 759

14 Jordan SE, Ahn SS, Gelabert HA. Combining ultrasonography and electromyography for botulinum chemodenervation treatment of thoracic outlet syndrome: comparison with fluoroscopy and electromyography guidance. Pain Physician 2007; 10: 541 - 546

15 Glass GA, Ku S, Ostrem JL et al. Fluoroscopic, EMG-guided injection of botulinum toxin into the longus colli for the treatment of anterocollis. Parkinsonism Relat Disord 2009; 15: 610-613

16 Garcia Ruiz PJ, Perez Higueras A, Quiñones D et al. Posterior CT guided approach for botulinum toxin injection into spinal psoas. J Neurol 2003; 250: 617-618

17 Stenner A, Reichel G, Hermann W. Successful treatment of piriformis syndrome with botulinum toxin A [abstract]. ] Neurol 2006; 253 (Suppl. 02): II/110

18 Reichel G. Cervical dystonia: A new phenomenological classification for botulinum toxin therapy. Basal Ganglia 2011; 1: 5-12

19 Mashayekh A, Christo PJ, Yousem DM et al. CT-guided injection of the anterior and middle scalene muscles:

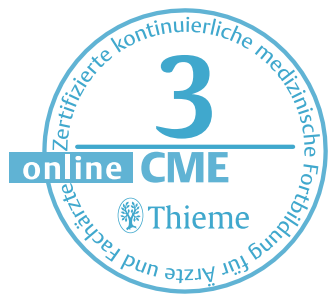


technique and complications. AJNR Am 」 Neuroradio 2011; 32: 495-500

20 Mezaki T, Matsumoto S, Sakamoto T et al. Cervical echomyography in cervical dystonia and its application to the monitoring for muscle afferent block (MAB). Rinsho Shinkeigaku 2000; 40: 689-693

21 Berweck S, Feldkamp A, Francke A et al. Sonography-guided injection of botulinum toxin $A$ in children with cerebral palsy. Neuropediatrics 2002; 33: $221-223$

22 Boon AJ, Oney-Marlow TM, Murthy NS et al. Accuracy of electromyography needle placement in cadavers: nonguided vs. ultrasound guided. Muscle Nerve 2011; 44: 45-49

23 Peetrons P. Ultrasound of muscles. Eur Radiol 2002; 12 $35-43$

24 Schmidt WA, Backhaus M, Sattler $\mathrm{H}$ et al. Imaging techniques in rheumatology: sonography in rheumatoid arthritis. Z Rheumatol 2003; 62: 23-33

25 Reimers CD, Fleckenstein JL, Witt TN et al. Muscular ultrasound in idiopathic inflammatory myopathies of adults. J Neurol Sci 1993; 116: 82-92

26 Reimers $C D$, Schlotter B, Eicke BM et al. Calf enlargement in neuromuscular diseases: a quantitative ultrasound study in 350 patients and review of the literature. J Neurol Sci 1996; 143: 46 - 56

27 Mayans D, Cartwright MS, Walker FO. Neuromuscular ultrasonography: quantifying muscle and nerve measurements. Phys Med Rehabil Clin N Am 2012; 23: 133 - 148

28 To EW, Ahuja AT, Ho WS et al. A prospective study of the effect of botulinum toxin $A$ on masseteric muscle hypertrophy with ultrasonographic and electromyographic measurement. Br J Plast Surg 2001: 54: 197-200

29 Stokes M, Hides J, Elliott J et al. Rehabilitative ultrasound imaging of the posterior paraspinal muscles. J Orthop Sports Phys Ther 2007; 37: $581-595$

30 English C. Fisher L, Thoirs K. Reliability of real-time ultrasound for measuring skeletal muscle size in human limbs in vivo: a systematic review. Clin Rehabil 2012; 26: 934 944

31 Alfen NV, Gilhuis HI, Keijzers JP et al. Quantitative facial muscle ultrasound: feasibility and reproducibility. Muscle Nerve 2013; 48: 375-380

32 Katz P, Hartl DM, Guerre A. Clinical ultrasound of the salivary glands. Otolaryngol Clin North Am 2009; 42: 973 1000

33 Tacik $P$, Dressler $D$. Ultraschallgestützte BotulinumtoxinInjektionen in der Neurologie. Klin Neurophysiol 2013; 44: $1-9$

34 Kopf H, Mostbeck GH, Loizides A et al. Ultrasound-guided interventions at peripheral nerves: diagnostic and therapeutic indications. Ultraschall Med 2011; 32: 440 -456

35 Walter U, Dressler $D$. Ultrasound-guided botulinum toxin injections in neurology: technique, indications and future perspectives. Expert Rev Neurother 2014; 14: 923-936

36 Hobson-Webb $L D$, Boon AJ. Reporting the results of diagnostic neuromuscular ultrasound: an educational report. Muscle Nerve 2013; 47: 608-610

37 Schramm A, Bäumer $T$, Fietzek $U$ et al. Relevance of sonography for botulinum toxin treatment of cervica dystonia: an expert statement. J Neural Transm 2014: 10.1007/s00702-014-1356-2

38 Gervasio A, D'Orta G, Mujahed I et al. Sonographic anatomy of the neck: The suprahyoid region. J Ultrasound 2011: 14: 130-135

39 Chaukar DA, Sayed SI, Shetty NS et al. Ultrasound-guided botulinum toxin injection: A simple in-office technique to improve tracheoesophageal speech in postlaryngectomy patients. Head Neck 2013; 35: E122 - 125

40 Blitzer A. Spasmodic dysphonia and botulinum toxin: experience from the largest treatment series. Eur \ Neurol 2010; 17 (Suppl. 01): $28-30$

41 Reichel G. Therapieleitfaden Spastik - Dystonien. Bremen: UNI-MED; 2012: 160
42 Gervasio A, Mujahed I, Biasio A et al. Ultrasound anatomy of the neck: The infrahyoid region. J Ultrasound 2010; 13: $85-89$

43 Heinen $F$, Desloovere $K$, Schroeder AS et al. The updated European Consensus 2009 on the use of Botulinum toxin for children with cerebral palsy. Eur J Paediatr Neurol 2010; 14: 45-66

44 Westhoff B, Seller K, Wild A et al. Ultrasound-guided botulinum toxin injection technique for the iliopsoas muscle. Dev Med Child Neurol 2003; 45: 829-832

45 Sconfienza LM, Perrone N, Lacelli $F$ et al. Ultrasound-guided injection of botulinum toxin $A$ in the treatment of iliopsoas spasticity. J Ultrasound 2008; 11: 113-117

46 Lee IH, Yoon YC, Sung DH et al. Initial experience with imaging-guided intramuscular botulinum toxin injection in patients with idiopathic cervical dystonia. AJR Am J Roentgenol 2009; 192: 996-1001

47 Javanshir K, Mohseni-Bandpei MA, Rezasoltani A et al. UItrasonography of longus colli muscle: A reliability study on healthy subjects and patients with chronic neck pain. J Bodyw Mov Ther 2011; 15: 50-56

48 Fujimoto $\mathrm{H}$, Mezaki $T$, Yokoe $M$ et al. Sonographic guidance provides a low-risk approach to the longus colli muscle. Mov Disord 2012; 27: 928 -929

49 Hong JS, Sathe GG, Niyonkuru C et al. Elimination of dysphagia using ultrasound guidance for botulinum toxin injections in cervical dystonia. Muscle Nerve 2012; 46: 535-539

50 Picelli A, Lobba D, Midiri A et al. Botulinum toxin injection into the forearm muscles for wrist and fingers spastic overactivity in adults with chronic stroke: a randomized controlled trial comparing three injection techniques. Clin Rehabil 2014; 28: 232-242

51 Santamato A, Micello MF, Panza F et al. Can botulinum toxin type $\mathrm{A}$ injection technique influence the clinical outcome of patients with post-stroke upper limb spasticity? A randomized controlled trial comparing manual needle placement and ultrasound-guided injection techniques. J Neurol Sci 2014; 347: 39-43

52 Picelli A, Tamburin S, Bonetti $P$ et al. Botulinum toxin type A injection into the gastrocnemius muscle for spastic equinus in adults with stroke: a randomized controlled trial comparing manual needle placement, electrical stimulation and ultrasonography-guided injection techniques. Am J Phys Med Rehabil 2012; 91: 957-964

53 Lim EC, Quek AM, Seet RC. Accurate targeting of botulinum toxin injections: how to and why. Parkinsonism Relat Disord 2011; 17 (Suppl. 01): S34-39

54 Dogu O, Apaydin D, Sevim S et al. Ultrasound-guided versus 'blind' intraparotid injections of botulinum toxin-A for the treatment of sialorrhoea in patients with Parkinson's disease. Clin Neurol Neurosurg 2004; 106: 93 -96

55 Porta M, Gamba M. Bertacchi $G$ et al. Treatment of sialorrhoea with ultrasound guided botulinum toxin type $A$ injection in patients with neurological disorders. J Neurol Neurosurg Psychiatry 2001; 70: 538-540

56 Turk-Gonzales M, Odderson IR. Quantitative reduction of saliva production with botulinum toxin type $B$ injection into the salivary glands. Neurorehabil Neural Repair 2005; 19: 58-61

57 Breheret R, Bizon A, Jeufroy $C$ et al. Ultrasound-guided botulinum toxin injections for treatment of drooling. Eur Ann Otorhinolaryngol Head Neck Dis 2011; 128: 224 229

58 Jeung IS, Lee S, Kim HS et al. Effect of botulinum toxin a injection into the salivary glands for sialorrhea in children with neurologic disorders. Ann Rehabil Med 2012; 36: $340-346$

59 Chan KH, Liang C, Wilson P et al. Long-term safety and efficacy data on botulinum toxin type A: an injection for sialorrhea. JAMA Otolaryngol Head Neck Surg 2013; 139: $134-138$

60 Giovagnorio F, Pace F, Giorgi A. Sonography of lacrimal glands in Sjögren syndrome. J Ultrasound Med 2000; 19: 505-509 


\section{CME-Fragen Botulinumtoxin-Therapie in der Neurologie: Indikationen, Technik und Ultraschallsteuerung}

1 Welche Aussage zur Anwendung des Ultraschalls zur Injektionssteuerung in den Muskel trifft zu?

A Das oberflächliche Muskelgewebe ist optimal mit einer Sondenemissionsfrequenz von 2,5 $\mathrm{MHz}$ darstellbar.

B Die übliche Injektionsnadel wird als heller Strich im B-Bild sichtbar.

C Zur Darstellung des Muskelgewebes im Ultraschall wird das B-Bild genutzt.

D Die Injektionsflüssigkeit kommt nur mit speziellen Kontrastmitteln im Ultraschall zur Darstellung.

E Der M-Mode im Ultraschall ist nur für die Bewegungsanalyse der Herzklappen geeignet.

2 Welche Aussage zur BT Injektion trifft nicht zu?

A Alle Armmuskeln sind für die intramuskuläre BT-Injektion geeignet.

B Der Zeitaufwand für die Ultraschallbenutzung ist vernachlässigbar.

C Zur BT-Behandlung des Schreibkrampfes wird die ultraschallgesteuerte BT-Injektion empfohlen.

D Im Ultraschall-B-Bild kann eine Dokumentation des Injektionsortes und der Lage des Injektionsvolumens erfolgen.

E Im Ultraschall-Farbduplex-Mode werden zusätzliche, benachbarte Strukturen wie Gefäße dargestellt.

Welche Aussage zur BT-Behandlung trifft nicht zu?

A Die ultraschallgesteuerte BT-Injektion wird zur Behandlung der Spastik bei Kindern mit Zerebralparese empfohlen.

B Die ultraschallgesteuerte BT-Injektion ist die empfohlene Behandlungsmethode zur Injektion in den M. omohyoideus.

C Die ultraschallgesteuerte BT-Injektion zur Behandlung der zervikalen Dystonie (M. scalenus anterior) wird nicht empfohlen, da als Nebenwirkung eine versehentliche Affektion des Plexus brachialis möglich ist.

D Klinische Studien zeigen eine Verbesserung der Trefferquote für BT-Injektionen in den M. gastrocnemius unter Ultraschallkontrolle im Vergleich zur konventionellen Methode.

E Eine EMG-gesteuerte BT-Injektion zur Behandlung des M. vocalis bei spasmodischer Dysphonie ist besser geeignet als eine ultraschallgesteuerte Injektion.

4 Welche Aussage für die Behandlung der exokrinen Drüsen mit BT trifft nicht zu?

A Die lokale Applikation von BT wird zur Behandlung der Hypersalivation eingesetzt.

B Durch die ultraschallgesteuerte BT-Injektion zur Reduktion der Hypersalivation werden Nebenwirkungen geringer.

C Die ultraschallgesteuerte BT-Injektion in die Glandula submandibularis wird empfohlen.

D Die ultraschallgesteuerte BT-Injektion in die Glandula parotis ist sinnvoll.

E Zur BT-Injektion in die Glandula lacrimalis ist die Ultraschallkontrolle notwendig.

\section{5}

A Zur Reduktion der Hypersalivation ist die EMG-gesteuerte BT-Injektion geeignet.

B Die BT-Injektion ist zur Behandlung der Spastik an den unteren Extremitäten nicht zugelassen.

C Die intramuskuläre BT-Injektion verändert die Muskelfunktion irreversibel.

D Sehr tief gelegene Muskeln ( $>6 \mathrm{~cm}$ unter Hautoberfläche) sind im Allgemeinen ungeeignet für die ultraschallgesteuerte BT-Injektion.

E Die intramuskuläre BT-Behandlung hat als rechtfertigende Indikation nur die Funktionsverbesserung.

6 Welche Aussage zur Behandlung mit BT trifft zu? Die ultraschallgesteuerte BT-Injektion wird empfohlen für...

A den M. pterygoideus.

B die mimische Muskulatur.

C die Pharynxmuskeln.

D die Larynxmuskeln.

E den M. longissimus capitis.

7 Welche Aussage zur BT-Behandlung von exokrinen Drüsen trifft nicht zu?

A Die Glandula sublingualis und lacrimalis sind im Ultraschall gut darstellbar.

B Die EMG-Methode zur Lokalisation des Injektionsortes ist für die Behandlung der exokrinen Drüsen nicht geeignet.

C Die Hyperhidrose ist eine Indikation für die BT-Injektion und bedarf keiner Ultraschallkontrolle.

D Die Behandlung der Krokodilstränen ist dem Veterinärmediziner vorbehalten.

E Eine gefürchtete Nebenwirkung bei BT-Injektionen in die Speicheldrüsen sind Schluckstörungen. 
8 Welche Aussage zur ultraschallgesteuerten BT-Injektion trifft nicht zu?

A Die Ultraschallmethode ist ein nicht invasives Verfahren und geeignet, Untersuchungen in Echtzeit durchzuführen.

B Die Desinfektion der Haut vor Anwendung des Ultraschalls erfolgt mit den üblichen Desinfektionsmitteln.

C Für die genaue Lokalisation der Nadelspitze vor Injektion ist die Applikation von Ultraschall-Kontrastmittel nicht erforderlich.

D Im Ultraschall-Duplex-Mode werden B-Bild und farbkodierte Flusssignale gleichzeitig dargestellt.

E Die Ultraschallmethode kann prinzipiell auch zur Injektionskontrolle von Lokalanästhesien eingesetzt werden.
9

A Blepharospasmus

B Bruxismus

C Hypersalivation bei Morbus Parkinson

D akuter Migräneanfall

E Schreibkrampf

\section{0}

Welcher Muskel stellt keine Herausforderung bei der exakten Anzielung (ohne Ultraschall oder EMG) dar in der BT-Behandlung der Spastik am Unterarm?

A M. flexor carpi radialis

B M. pronator teres

C M. biceps brachii

D M. flexor pollicis longus

E M. flexor digitorum superficialis II/III 